\title{
Du corps altéré au corps sportif $\stackrel{\infty}{\stackrel{\infty}{\Phi}}$.
}

\author{
Jean-Pierre GAREL \\ Laboratoire Relacs, Ulco \\ Université du Littoral Côte d'Opale
}

Résumé: Tout sépare a priori un corps altéré d'un corps sportif, au point qu'il peut être difficilement pensable de favoriser l'accès de jeunes présentant une déficience physique à une pratique sportive. II s'agit de montrer que les représentations communes sous-estiment grandement leurs possibilités et qu'ils peuvent tirer de cette pratique des bénéfices définis en termes d'accomplissement personnel et de lien social, en y trouvant notamment matière à une dynamique identitaire positive. L'école ayant à jouer un rôle déterminant dans l'accès de tous les élèves à ces activités, des conditions sont envisagées pour que des jeunes dont le corps est altéré soient en mesure de le mobiliser avec profit en EPS.

Mots-clés: Accomplissement - Corps - Éducation physique - Estime de soi - Handicap - Identité - Participation sociale - Plaisir (du sport) - Sport.

\section{From distored body to sports body}

Summary: Everything separates a priori a body distorted by a sports body, to the point that it can be hardly thinkable to facilitate the access of young people with physical disabilities in sports. It is a question of showing that the common representations greatly underestimate their potential and they can get out of this practice benefits defined in terms of personal fulfillment and social link, particularly in regard to finding a dynamic positive identity. The school having to play a decisive role in the access of all the pupils to these activities, conditions are envisaged so that young people whose body is distorted are able to mobilize it with profit in physical education.

Key words: Body - Fulfillment - Identity - Physical education - Pleasure - Self-esteem - Social participation Sport.

$\mathbf{U}$ NE personne qui présente une déficience physique ne correspond pas à l'image d'un corps sain, beau et performant que l'on se fait souvent du sportif, et les difficultés qu'elle peut rencontrer au quotidien dans certaines situations impliquant l'usage de son corps ne laissent pas spontanément imaginer sa capacité à le mobiliser avec succès lors d'une pratique sportive. De fait, cette pratique est bien moindre chez les personnes en situation de handicap que chez les valides (Marcellini, Banens, 2003).

Notre propos nécessite de préciser ce que nous entendons ici par le terme de sport. Au sens restreint qui le limite à des activités physiques pratiquées dans un cadre institutionnalisé, au sein de clubs rattachés à une fédération sportive, nous préférerons une définition plus large, qui étend son domaine aux activités physiques " libres ", pratiquées avant tout dans un objectif de bien-être ou de convivialité, et à celles qui sont pratiquées à l'école, appelées dans les textes officiels de l'Éducation nationale " activités physiques, sportives et artistiques ". Dans cette perspective, il apparaît que, si le sport appelle souvent des efforts pour se dépasser et viser 
I'excellence, il n'implique pas obligatoirement la compétition avec autrui : le joggeur du dimanche, le nageur de l'été et celui qui s'adonne à la danse ou aux arts du cirque sont bien des "sportifs".

L'activité sportive peut être bénéfique, sous réserve qu'elle soit suffisamment raisonnée pour ne pas verser dans des excès qui mettent à mal l'intégrité physique, psychologique et morale de l'individu. Dès lors se pose la question de savoir comment permettre à des personnes présentant une déficience physique de s'y livrer pour en retirer les bénéfices attendus. Parmi les obstacles qu'elles peuvent rencontrer pour y accéder, on retiendra des représentations qui s'attachent communément aux corps altérés et dont le sens péjoratif marque une distance, entre ce type de corps et celui du sportif, telle qu'il peut être difficilement pensable de la réduire et d'envisager une pratique sportive par des personnes en situation de handicap. D'un côté, on aurait un corps impuissant, caché, souffrant, qui isole, etc., et de I'autre un corps performant, exposé, source de plaisir, qui réunit... II s'agit d'effacer la ligne séparant ces "colonnes des contraires " (Bourdieu, 1980, p. 348-349), de déconstruire ce système d'oppositions propre à paralyser l'action éducative, et d'envisager quelques conditions, d'ordre pédagogique, pour que l'école permette aux jeunes dont le corps est altéré de le mobiliser avec profit en EPS.

\section{DU SENTIMENT D'UN CORPS IMPUISSANT AU CORPS PERFORMANT}

La difficulté, voire l'incapacité, de mobiliser son corps varie selon la déficience, les individus et les situations qu'ils rencontrent, mais pour tous ceux qui sont ainsi entravés dans leur désir et leur pouvoir d'agir c'est une source de frustrations, le fondement d'un sentiment d'impuissance qui peut dépasser la sphère corporelle pour affecter le sujet dans toutes ses dimensions. Le retentissement d'une déficience trouve à s'illustrer dans la parole d'Élodie, six ans, atteinte d'une Infirmité motrice cérébrale (IMC), à qui un adulte demande de s'expliquer sur ce qu'elle vient de lui dire: "quand on ne peut pas marcher on n'est bon à rien ". Réponse de l'enfant: " on ne peut pas aider maman... " (Truscelli, 2008, p. 394). Concernant les adolescents IMC, Éliane Gérard avait déjà noté la prédominance d'un sentiment d'impuissance qui s'enracine dans le corps et se répand ailleurs (1991).

Dans le cas de jeunes présentant une atteinte neuromusculaire évolutive, les conséquences psychologiques d'une incapacité physique croissante, qui se manifeste notamment par la perte de la marche, sont également signalées. Hervé Benony décrit comme symptômes fréquents " une inhibition parfois massive, fantasmatique et/ou relationnelle, le désinvestissement du monde extérieur ou scolaire et la dépression " (1986).

Du constat d'indéniables limitations motrices et de la présentation par différents auteurs du sentiment d'impuissance qui les accompagne, il ne faut pas tirer des conclusions qui réduiraient les personnes à un portrait-type particulièrement pessimiste, dressant un fossé infranchissable entre ceux dont le corps est altéré, incapable, et ceux qui sont désignés comme "sportifs". La singularité de chaque individu met en défaut les affirmations globalisantes. D'autres raisons d'envisager l'accès à des activités jugées a priori hors de portée tiennent à la conscience accrue qu'il n'y a pas de handicap en soi, indépendamment des situations vécues et des influences 
éducatives, et au pari d'une éducabilité qui se fonde sur une meilleure connaissance - et sur sa prise en compte - des capacités d'individus dont on a longtemps retenu que les manques. Aujourd'hui, de jeunes aveugles jouent au basket-ball; d'autres, IMC ou myopathes, pratiquent en fauteuil à propulsion électrique un football adapté qui donne lieu à des compétitions nationales et internationales...

Une pratique sportive de loisir, hors de toute compétition, peut aussi être l'occasion $d^{\prime}$ incontestables performances. Patrice Radiguet, "pour le plaisir, tout simplement ${ }^{1}$ " et malgré une acuité visuelle de moins de $1 / 10^{e}$, pratique, ou a pratiqué, la spéléologie, l'escalade, le ski, le judo, le canoë, le VTT, le pilotage d'avions de tourisme et l'initiation à la voltige aérienne... Un bain de mer est un loisir plus modeste, mais surprenant quand il est le fait d'une personne aveugle qui ose en toute autonomie nager en eau profonde, grâce à un aménagement fournissant des informations tactiles et sonores qui lui permettent de localiser des bouées et d'évaluer les distances ainsi que la profondeur de l'eau ${ }^{2}$. On voit là l'importance du contexte.

Sans aménagement particulier, un environnement peut s'avérer propice à une mise en jeu corporelle gratifiante. Alexandre Jollien évoque une jeune fille "rayonnante " à la piscine. Elle avait vécu en Afrique dans un pays en guerre, où des soldats I'avaient laissée les bras et les jambes coupés: "paisiblement, elle nageait sur le dos.[...] À la regarder flotter, avec son sourire éternel, elle incarnait à mes yeux la joie la plus totale, une joie unique. Merveilleuse faculté d'adaptation de l'homme " (2003, p. 55). L'eau est effectivement un milieu qui permet à des personnes présentant des restrictions motrices très invalidantes d'évoluer avec davantage de facilité.

On peut aussi parler de performance s'agissant d'artistes mettant en scène leur corps déficient de façon étonnante, par exemple dans le cas de l'américain Bill Shannon, qui se déplace ordinairement à l'aide de béquilles. C'est un danseur et chorégraphe réputé, surnommé The Crutch Master (le maître des béquilles), récompensé par plusieurs prix et distinctions, et boursier de la Foundation for Contemporary Performance Art.

En dehors d'une pratique sportive proprement dite, une personne peut mobiliser au quotidien un corps sévèrement altéré dans un esprit de dépassement souvent avancé comme étant une caractéristique essentielle du sport. Alexandre Jollien, atteint d'une infirmité motrice cérébrale, en est une illustration. II se souvient de la " myriade d'exercices " auxquels il se livrait: " je m'entraînais à m'asseoir correctement, à coordonner mes jambes et mes bras. [...] J'essayais enfin quotidiennement d'améliorer mon résultat du mille mètres à quatre pattes. [...] Lutter envers et contre tout: telle était notre maxime, lutter contre l'immobilisme de certains éducateurs, lutter contre le diagnostic médical, contre le découragement. "L'activité sportive n'était pas étrangère à son désir et à sa lutte: "le médecin [...] décréta le vélo "impossible." Au mépris du diagnostic médical, je parvins à tenir sur deux roues. [...] II faut toujours se dépasser, sans cesse aller au-delà de soi-même, s'engendrer, parfaire ce qui est déjà réalisé en soi » (ibid., p. 15-58).

\footnotetext{
1. Titre d'une conférence de Patrice Radiguet lors du troisième congrès européen du Groupement de recherche et d'information consacré à la cécité et à l'amblyopie, à Bruxelles, en novembre 1997.

2. http://www.ile-oleron-marennes.com/utilitaires/handioleron.html
} 
Le sens de "parfaire " fait écho à celui de "performance ", puisque les deux termes contiennent l'idée d'accomplissement. Par la volonté de s'accomplir, des sujets arrivent à transcender plus ou moins l'impuissance à laquelle ils semblaient destinés, avec autant que possible l'aide de leur entourage et des professionnels intervenant auprès d'eux pour les accompagner dans cet effort. L'école participe fondamentalement à cette conquête. Par ce que l'enseignement de l'éducation physique qui y est dispensé s'adresse normalement à tous, il donne à des jeunes en situation de handicap l'occasion d'acquérir ou de développer des capacités, des compétences et des attitudes dont témoignent des films produits par l'INS HEA ${ }^{3}$.

\section{CORPS CACHÉ, CORPS EXPOSÉ}

Lorsqu'elles rencontrent des personnes dont les altérations corporelles sont manifestes, les personnes valides ressentent une gêne perçue par ceux qui sont ainsi l'objet de regards troublés. D'où, pour Jean-Luc Simon, paraplégique, le " malaise de se trouver au milieu d'autres personnes qui risquent de vous regarder " (1991). Un malaise, selon lui, " qui fait référence à l'interdiction d'exposer son corps devant les autres (à la piscine ou à la plage par exemple) » (ibid.). L'interdit est en fait celui que s'inflige un individu qui a intégré une image de son corps socialement indésirable. "Au Centre, écrit Alexandre Jollien à propos de son enfance, le corps restait caché. Par lui-même, il constituait en quelque sorte un tabou, non pas entre nous, mais en général, la politique de la maison ne privilégiait pas un sain contact avec le corps » (ibid., p. 73).

La prégnance de représentations valorisant les corps sains, minces, jeunes, musclés, qui répondent à des critères esthétiques contraignants, et qui symbolisent la performance que notre société a érigée en objet de culte, est lourde d'effets stigmatisant sur les personnes éloignées de ces références. Lorsqu'on présente une déficience physique, il est alors difficile d'envisager de s'exposer dans des activités physiques à caractère artistique. Avant même de se montrer dans un spectacle, les regards extérieurs peuvent être une épreuve lors des séances préparatoires. Ainsi Céline, IMC, confie-t-elle: "Je me suis mise à pleurer. J'avais une angoisse. II fallait que je montre mon travail et pour moi c'était dur de le montrer, que les autres regardent. Je ne me sentais pas à l'aise. J'avais peur de ne pas réussir " (Bouquet des Chaux, 1996, p. 94).

Pourtant, les expressions sportives d'un corps altéré peuvent être regardées positivement, notamment parce qu'aujourd'hui "la relation de l'individu à son corps se fait sous l'égide de la maîtrise de soi, I'homme est invité à construire son corps, façonner son apparence, occulter le vieillissement ou la fragilité " (Le Breton, 1999, p. 26). La valeur accordée au contrôle de soi exercé sur son corps symbolise la capacité à contrôler sa vie. Elle est congruente à l'exaltation actuelle de l'individu autonome, "entrepreneur de soi-même ". Selon cette logique, ce sont moins les caractéristiques et les qualités physiques qui importent que "les efforts accomplis

3. Voir notamment Éducation physique et sportive et déficience motrice au lycée Toulouse-Lautrec et Activités physiques et sportives avec des élèves aveugles. 
par les individus afin de modifier leur corps et acquérir ainsi par la volonté les caractéristiques requises par la société " (Parisoli, ibid., p. 22).

Le regard porté en EPS sur l'enfant ou l'adolescent présentant une déficience physique va dans ce sens. Des études conduites dans des collèges accueillant des élèves de ce type montrent qu'ils apprécient les cours d'EPS avec leurs camarades ordinaires, et qu'en retour leurs altérations ne suscitent pas de rejet (Garel, 1999). Au contraire, elles forcent l'admiration pour les performances qu'elles autorisent, relatives certes mais parfois surprenantes, et pour l'engagement et la persévérance de ceux qui en sont atteints et qui, de ce fait, constituent un modèle pour leurs camarades ${ }^{4}$. Ainsi, en témoignant d'efforts pour dominer un corps rétif, ces collégiens singuliers se donnent-ils à voir sous un jour positif.

Si l'on considère la place revendiquée et croissante, bien qu'insuffisante, des personnes en situation de handicap dans les espaces de vie ordinaires, à l'école et ailleurs, on peut en déduire que l'appréhension des regards extérieurs diminue et faire I'hypothèse qu'elle diminuera encore, au fur et à mesure que l'habitude de vivre ensemble dès l'enfance fera apparaître l'autre moins étrange que familier.

\section{CORPS QUI ISOLE, CORPS QUI RÉUNIT}

Dès lors qu'une image de soi dévalorisée n'incite pas à s'exposer au regard des autres, elle risque de faire obstacle à des loisirs partagés, comme pour ce jeune, atteint d'une maladie neuromusculaire, qui exprime sa crainte de "gêner les autres, de n'être perçu par l'entourage potentiel que de façon négative " (Sanchez et Bourderon, 1990). Lorsque la confrontation au handicap survient brutalement, à la suite d'un accident, la personne se trouve exclue des groupes dont elle faisait partie, au moins pendant le temps de l'hospitalisation. Jean-Luc Simon avance que l'exclusion de son club sportif, comme éventuellement de son travail, "contribue au dépouillement de son identité; en plus de la perte d'identité corporelle, son identité groupale s'évapore aussi " (ibid.). II ajoute que le contact et l'identification avec d'autres dans une situation analogue sont une étape indispensable après I'hospitalisation, bien que difficile à accepter.

Le regroupement avec des semblables peut alors devenir " un choix, et une rencontre en miroir " (Marcellini, 2005, p. 92), le groupe de pairs s'offrant " comme un espace de déstigmatisation "interne," dans le sens où l'attribut stigmatisant y devient la norme. Il devient de ce fait espace de "repos," sécurisant, chaleureux, espace social sans regard stigmatisant " (ibid., p. 103), où chacun " porte le regard sur le corps des autres et y recherche les traits identificatoires qui lui manquent " (Missenard, 1982, p. 27). Une activité sportive au sein d'un groupe composé de personnes présentant un même type de déficience, par exemple dans le cadre d'une fédération qui leur est spécifique, contribue à forger une identité commune. Elle est particulièrement pertinente si l'on considère qu'une personne handicapée participera d'autant plus volontiers à l'activité d'un groupe de pairs qu'elle aura l'occasion d'y " montrer son pouvoir d'action, [...] qu'elle pourra y faire preuve de son intégrité retrouvée, [...] montrer que la déstructuration et la perte figurées par le corps déformé, angoisses

4. Voir par exemple le film Une UPI pour élèves présentant une déficience motrice, produit par I'INS HEA. 
de tous les membres à leur arrivée dans le groupe, peuvent être générateurs de re-naissance et de maîtrise " (Simon, ibid.).

Les aspects positifs d'une identification aux personnes partageant une situation de handicap semblable ne sont pas propres aux personnes qui ont été accidentées ou qui présentent une déficience motrice (Murphy, 1990, p. 220). Mais tous les individus ne préfèrent pas vivre entre soi. Pour Jérôme par exemple, 16 ans, aveugle congénital, le regroupement avec des pairs aveugles ne convient pas. Les camarades voyants sont omniprésents dans son discours et il dit s'ennuyer avec les autres non voyants (Lewi-Dumont, 1999, p. 72-73).

Les personnes handicapées sont de plus en plus nombreuses à accéder aux espaces de vie ordinaire, à l'école, dans les loisirs, etc., et à s'y maintenir parce qu'elles y trouvent plus ou moins matière à satisfaction. Questionnés sur les séances d'EPS qu'ils préfèrent, entre celles où ils sont avec leurs camarades de classe, valides, et celles regroupant les élèves du collège dans la même situation qu'eux, des collégiens en situation de handicap choisissent les premières, ou bien ils témoignent d'une ambivalence (Garel, 1999, 2005) caractéristique d'une identité individuelle plurielle, construite à partir de multiples appartenances, ou identités collectives - " nous les fauteuils ", "nous les collégiens ", "nous les basketteurs "...-qui invitent chacun à conjuguer ses différences et ses ressemblances dans un itinéraire de vie singulier. Même limitée à une pratique entre soi, une activité sportive est un facteur de participation sociale si elle est l'occasion de sortir de chez soi, du lieu de vie et de scolarisation habituel, d'élargir son environnement physique et humain en ouvrant au sujet la possibilité de s'inscrire dans un réseau de sociabilité. L'enseignement de l'EPS doit donc prendre en compte la pertinence éventuelle d'une activité entre pairs. Si la participation des élèves en situation de handicap aux cours avec leurs camarades valides doit être prioritairement recherchée, certains d'entre eux gagnent à être parfois regroupés.

\section{DU CORPS DOULOUREUX AU PLAISIR DU CORPS : ENJEUX IDENTITAIRES}

Soumis à des soins et à des rééducations, le corps des jeunes présentant une déficience motrice est source de douleurs physiques pouvant s'accompagner d'une souffrance psychologique, particulièrement dans le cas d'une atteinte évolutive, qui accroît progressivement la dépendance. Devenant de plus en plus un objet de manipulation et de surveillance, l'individu risque de verser dans une passivité induite par un sentiment d'impuissance et par l'absence de plaisir retiré de l'activité (Réveillère, 1994). Dans ces conditions, la pratique d'activités sportives ne va pas de soi, comme, pour d'autres raisons, dans le cas de blessés médullaires. Pris par le déni de leur handicap, constaté " à travers la projection défensive d'une image du corps intacte, d'une représentation de soi idéale " (Bilard, 1993), certains d'entre eux évitent les situations susceptibles de les confronter à la réalité, de raviver la conscience de pouvoirs disparus.

Cela dit, une déficience physique, même sévère, ne retient pas fatalement l'engagement dans une activité sportive et, à travers elle, la recherche de plaisirs. Françoise de Barbot parle de " jubilation " pour décrire ce qu'éprouvent des enfants 
IMC, dont aucun ne marche, lors d'activités motrices liées au cirque, et elle regrette que lorsque des adolescents IMC demandent de faire du sport, de danser, trop souvent les adultes comprennent cette demande comme une négation du handicap. "Ne devrait-on pas y reconnaître d'abord, demande-t-elle, la recherche d'un plaisir rarement offert ? [...] Ne devrait-on pas chercher à préserver cet investissement fragile d'une activité corporelle? " (1987, p. 221).

Éliane Gérard, qui a souligné le sentiment d'impuissance ressenti par des adolescents IMC, note, paradoxalement, une "inflation prodigieuse " du thème " jeux et sports " dans leurs récits spontanés. L'image de soi comme incapable n'entrave pas l'expression d'un désir de mise en jeu corporelle dans des activités sportives. Selon Annie Birraux, le corps stigmatisé est un corps de désir qui, de ce point de vue, n'a rien à envier à un corps intègre (1994, p. 150). Plus généralement, une infirmité n'empêche pas les gens d'avoir une vie heureuse et pleine (Parisoli, ibid., p. 65) ; une assertion également soutenue par Alexandre Jollien, qui avance que ce n'est pas parce que l'on souffre que l'on est malheureux et que les personnes en situation de handicap lui enseignent à " jubiler devant la vie » (2002, p. 54).

Un plaisir que des personnes présentant une déficience motrice disent ressentir lors d'une pratique sportive est de l'ordre des sensations. Des sensations dues par exemple à l'effort, à la dépense d'énergie, au contact avec l'environnement naturel, aux évolutions dans l'eau, particulièrement pour ceux dont la déficience est sévère, puisqu'ils n'y sont pas soumis aux mêmes contraintes de pesanteur que sur terre. Le plaisir ressenti diffuse en dehors de la sphère sensorimotrice : réussir un "beau geste ", marquer un "beau point ", dominer sa peur, dépasser ses limites... (Turpin et al., 2005). Ce qui est éprouvé " dans la mise en jeu physique [...] entraîne le sentiment fort d'exister » (Le Breton, 1990, p. 128) et peut conduire à une évolution identitaire: " maintenant quand je danse, je suis quelqu'un d'autre ", confie une adolescente présentant une déficience motrice (Garel, 2002, p. 99). Cette évolution se comprend à l'écoute de personnes se disant "étonnées ", "impressionnées ", par ce qu'elles arrivent à réaliser avec leur corps (ibid.).

La pratique d'une activité sportive n'est pas que corporelle. C'est le sujet dans sa globalité qui s'y implique et dont le pouvoir d'agir se trouve reconnu dans les résultats qu'il obtient; reconnu par lui-même et par les autres, indissociablement, car "la reconnaissance de soi comme homme "capable" de certains accomplissements [...] requiert I'aide d'autrui " (Ricœur, 2004, p. 109). Cette reconnaissance contribue à fonder l'identité, puisqu'une caractéristique de l'identité est liée à l'estime de soi, et que "I'identité renvoie alors à l'idée de réalisation de soi par l'action, du "devenir soi-même" à travers des activités (faire, et en ce faisant, "se faire") " (Tap, 1996, p. 9).

Dans la perception que I'on a d'une personne en situation de handicap, le handicap tend à prendre le pas sur les autres dimensions de l'identité, du fait que "l'invalidité exerce sur le sens de qui on est, de ce qu'on est, une emprise bien plus forte que n'importe quel rôle social " (Murphy, ibid., p. 150). En montrant, lors de l'activité sportive, des capacités et des rôles qui ébranlent les préjugés, celui dont le corps est altéré diminue la probabilité de n'être vu qu'à travers le prisme de son handicap. 


\section{LA MOBILISATION CORPORELLE EN EPS COMME FACTEUR D'ACCOMPLISSEMENT PERSONNEL ET DE LIEN SOCIAL... SOUS CONDITIONS}

Au terme de notre comparaison entre un corps altéré et un corps sportif, il apparaît que les oppositions radicales qui pouvaient a priori les caractériser cèdent à l'analyse. On peut avancer que, engagées dans une activité sportive, les personnes présentant une déficience physique y sont capables de développer des pouvoirs d'agir significatifs, que cette activité est susceptible de créer ou resserrer le lien social, qu'elle est source de plaisir et fondatrice d'une identité qui s'extrait de l'enfermement dans un statut de handicapé. Un corps altéré peut devenir un corps sportif et cette évolution conduire le sujet à des transformations positives, qui ne se limitent pas au domaine physique.

L'école constitue un lieu privilégié pour satisfaire à ces enjeux d'accomplissement personnel et de participation sociale, dans la mesure où la mobilisation corporelle de I'élève en situation de handicap, à l'œuvre dans le cadre des cours d'EPS, rencontre des éléments favorables; plus précisément, pour ne retenir que quelques-uns d'entre eux, d'ordre pédagogique, dans la mesure, autant que possible:

- où l'élève n'est pas l'objet de jugements préétablis qui, I'enfermant dans des catégories supposées définir totalement ses caractéristiques ("les infirmes moteurs cérébraux sont... "), occultent sa singularité et ses potentialités, et entravent son évolution (Jollien, 2003, p. 31);

- où l'enseignant ne considère pas les personnes en situation de handicap comme des " patients " auxquels il convient de proposer des activités de nature rééducative; où, à l'opposé, il privilégie des activités physiques, sportives et artistiques qui relèvent d'une culture commune, qui sont des pratiques sociales valorisées et valorisantes, dont un enjeu est "I'instauration du lien social et des modalités d'identité qui s'y rattachent » (Ricœur, ibid., p. 202);

- où l'accessibilité des activités proposées est travaillée par des enseignants ouverts à la différenciation pédagogique et suffisamment compétents pour intervenir auprès de ce type d'élève (Garel, 2007);

- où la prise en compte des différences s'accompagne de la prise en compte des ressemblances, car si une personne en situation de handicap a des singularités - comme tout un chacun, à des degrés divers - elle partage avec les valides des caractéristiques semblables, en termes de besoins, de désirs, etc., qui invitent à ne procéder à des adaptations de l'enseignement qu'à hauteur de ce qu'il est nécessaire, sans en rabattre sur les ambitions que l'on peut nourrir à l'égard de cette personne (Garel, 2009);

- où les relations interindividuelles et la vie du groupe sont favorisées, et où, conjointement, I'autonomie de l'élève est recherchée (Jollien, ibid., p. 66), par la possibilité qui lui est donnée d'agir de lui-même, d'exercer son jugement, de s'exprimer, y compris pour solliciter une aide, de faire des choix relatifs aux projets le concernant et à la façon de résoudre les problèmes rencontrés lors de son activité; - où l'enseignant exprime aux jeunes en manque d'assurance sa confiance dans leurs possibilités (Jollien, ibid., p. 67); 
- où le jeune est valorisé, conduit à prendre conscience de ses réussites et à exposer ses "œuvres " aux regards extérieurs (Garel, 2006);

- où l'attention à valoriser un élève n'aboutit pas à le leurrer sur ses forces et ses faiblesses, à l'empêcher ainsi de se connaître et de s'accepter (Jollien, ibid., p. 5159), car mobiliser son corps dans une activité sportive accroît les capacités mais ne supprime pas les altérations et certaines de leurs conséquences;

- où la volonté de privilégier l'accueil en milieu ordinaire des jeunes en situation de handicap ne conduit pas à sous-estimer l'intérêt de regrouper parfois certains d'entre eux (Garel, 1999) ;

- où les activités suscitent chez le jeune un plaisir suffisant, parce que "l'efficacité intégrative de ces activités [...] implique le plaisir partagé des acteurs, sinon elles échouent au plus élémentaire en maintenant une ligne de démarcation entre les uns, porteurs de handicap et de stigmate, et qu'il faudrait "redresser," et les autres " (Le Breton, ibid.). Mais au-delà de la recherche d'un plaisir que l'on peut entendre comme la satisfaction achevée d'un besoin, il importe que le jeune investisse les activités d'un sens qui l'incite à s'y engager et s'y maintenir.

L'acquisition de pouvoirs d'agir, favorisée par les éléments énumérés, passe par la mobilisation de ce qui est à l'origine de l'incapacité: le corps. La (re)conquête de soi s'appuie sur ce qui fait défaut, la déstigmatisation sur le lieu du stigmate, ce qui témoigne de la capacité du sujet à maîtriser son destin davantage que prévu, souvent. En surpassant ainsi son handicap, il accroît le sentiment de sa valeur personnelle, à ses propres yeux et aux yeux d'autrui, et il engage une dynamique identitaire qui nourrit et se nourrit des appartenances rendues possibles par les pouvoirs acquis et les relations vécues avec les autres: participation à des groupes divers, formels ou informels, à l'école ou durant les loisirs, par exemple en s'affiliant à une (des) association (s) sportive (s) - scolaire(s), rattachée(s) ou non à une fédération dédiée aux personnes en situation de handicap. Des espaces de vie commune en milieu ordinaire s'ouvrent, pour autant qu'ils soient suffisamment accessibles.

\section{Bibliographie}

BOuQueT DES ChauX (B.), "Danser, un envol par-delà le handicap ", Marsyas, $n^{\circ}$ 39-40, p. 92-96, 1996.

DE BARBOT (F.), " Enfants handicapés moteurs: enfant malade? ", in P. FERRARI et R. LAZAROVICl, dir., L'enfant malade et son corps, Privat, Toulouse, 1987.

BENONY (H.), "Approche psychopathologique des adolescents myopathes ", Perspectives psychiatriques, $n^{\circ} 2$, p. 110-124, 1986.

BILARD (J.), "Le désir de rendre l'autre sportif ", Quel corps?, n 43-44, 1993. BIRRAUX (A.), L'adolescent face à son corps, Éd. Universitaires, Paris, 1994.

BOURDIEU (P.), Le sens pratique, Éd. de Minuit, Paris, 1980.

GAREL (J.-P.), "Individualiser pour réunir: I'enseignement de I'EPS devant un paradoxe de l'intégration scolaire ", La nouvelle revue de I'AIS, n 8, 1999, p. 153-165. 
GAREL (J.-P.), "Expressions corporelles à l'épreuve d'altérations du corps et des sens ", La nouvelle revue de I'AlS, n 18, p. 97-106, 2002.

GAREL (J.-P.), "Adolescents au corps altéré et apprentissages par corps ", La nouvelle revue de l'AIS, $n^{\circ}$ 29, p. 33-46, 2005.

GAREL (J.-P.), " La reconnaissance de la capacité d'agir sur et par un corps altéré, enjeu de la construction du sujet ", Éduquer, L'harmattan, p. 61-71, 2006.

GAREL (J.-P.), "Accessibiliser les pratiques sportives ", in C. GARDOU et D. POIZAT, Désinsulariser le handicap. Quelles ruptures pour quelles mutations culturelles? Toulouse, Érès, p. 223-231, 2007.

GAREL (J.-P.), " EPS et situations de handicap: projets personnalisés et activités communes", in F. BRUNET, C. BLANC, A.-C. MARGOT, Polyhandicap-Handicap sévère-Activités motrices et sensorielles, Actio, Joinville-le-Pont, p. 131-146, 2009. GÉRARD (É.), "Être infirme moteur cérébral », in R. PERRON, dir., Les représentations de soi: développements, dynamiques, conflits, Privat, Toulouse, 1991.

LEWI-DUMONT (N.), " Moi je n'ai pas envie de prendre ce qui reste: proclamation de l'identité et de l'altérité chez des adolescents déficients visuels ", La nouvelle revue de I'AIS, n 4, p. 66-77, 1999.

JOLLIEN (A.), Le métier d'homme, Seuil, Paris, 2002.

JolliEn (A.), Éloge de la faiblesse, Éd. du Cerf, Paris, 2003.

LE BRETON, (D.), Anthropologie du corps et modernité, Puf, Paris, 1990.

LE BRETON, (D.), L'adieu au corps, Métailié, Paris, 1999.

LE BRETON, (D.), "Viser I'excellence personnelle ", in C. GARDOU, D. POIZAT, Désinsulariser le handicap. Quelles ruptures pour quelles mutations culturelles? Érès, Toulouse, 2007.

MARCELLINI (A.), BANENS (M.), Pratiques sportives et personnes handicapées en France, Exploitation de l'enquête HID, Rapport final, Montpellier 1, 2003.

MARCELLINI (A.), Des vies en fauteuil, CTNERHI, diff. Puf, Paris, 2005.

MARZANO-PARISOLI (M. M.), Penser le corps, Puf, Paris, 2002.

MISSENARD (A.), "Du narcissisme dans les groupes ", in R. KAES et col., Le travail psychanalytique dans les groupes, Tome 2, Dunod, Paris, 1982.

MURPHY (R. F.), Vivre à corps perdu, Plon, Paris, 1990.

RÉVEILLÈRE (C.), "Approche clinique du vécu de la mort chez le jeune myopathe ", Perspectives psychiatriques, $\mathrm{n}^{\circ}$ 42, 1994.

SANCHEZ (J.), BOURDERON (P.), Conditions de vie et d'autonomie des sujets atteints de maladie neuromusculaire et des sujets souffrant de mucoviscidose dans un département, Rapport d'études, AFM, Évry, 1990.

RICCEUR (P.), Parcours de la reconnaissance, Stock, Paris, 2004.

SIMON (J.-L.), "L'image de soi. Incidence de la visibilité du handicap dans la relation à autrui et aux groupes ", in C. GARDOU, dir., Handicap handicapé, le regard interrogé, Éres, Toulouse,1991. En ligne sur http://jlsimon.free.fr/articleshtm/ publication/imsoi.html

TAP (P.), "Marquer sa différence ", Sciences humaines, Hors-série n 15, p. 9-10, déc. 1996.

TURPIN (J.-P.) et al., "Plaisir et handicap physique ", Corps et Culture, n 5, 2000.

En ligne sur: http://corpsetculture.revues.org/document389.html

TRUSCELLI (D.), dir., Les infirmités motrices cérébrales. Réflexions et perspectives sur la prise en charge, Masson, Paris, 2008. 